\title{
BUILDING SYNTHETIC SOCIAL NETWORKS USING ASSOCIATION RULES AND CLUSTERING METHODS: CASE STUDY ON GLOBAL TERRORISM DATABASE
}

\author{
Jan Górecki ${ }^{1}$, Kateřina Slaninová ${ }^{2}$ \\ ${ }^{1}$ Slezská univerzita, Obchodně podnikatelská fakulta, Univerzitní nám. 1934/3, 73340 Karviná \\ Email:gorecki@opf.slu.cz \\ ${ }^{2}$ Slezská univerzita, Obchodně podnikatelská fakulta, Univerzitní nám. 1934/3, 73340 Karviná \\ Email: slaninova@opf.slu.cz
}

\begin{abstract}
The authors of the paper present an approach for datamining methods combination (method for association rules extraction and clustering method are combined) which is used for synthetic social networks construction which afterwards represents potentially interesting relations in analyzed data. Described approach is feasible for general data, principles of described approach, examples and experiments are illustrated on a part of unique database, which contain data about terroristic attack committed on all over the World (Global Terrorism Database). Thus this paper also extend framework of papers focused on analysis of this unique database.
\end{abstract}

Keywords: association rules, community analysis, graph visualization, synthetic social network.

JEL classification: C38, Z00

Doručeno redakci: 22.6.2012; Recenzováno: 13.3.2013; 10.3.2013; Schváleno k publikování: 19.6.2013

\section{Introduction}

Data mining techniques like clustering methods and association rule mining methods are methods for the analysis of observational data sets used to find unsuspected relationships and to summarize the data in novel ways that are both understandable and useful to the data owner (Hand, 2001). Data mining is commonly a multistage process of extracting previously unanticipated knowledge from large data collections, and applying the results to decision making (Benoit, 2002). Data mining tools are able to detect patterns from the data and infer associations and rules from them. The development and the fundamental methods of the social network visualization were published by Freeman (2004). The extracted information can be applied in the prediction process or for construction of classification models using the relations within the data records or between the data collections. Those patterns, in Social network analysis, can be represented as patterns of groups (or communities) in social network structure and can then guide the network visualization and the study of network evolution. This type of information can be valuable in the decision making process and forecast the effects of those decisions.

The main purpose or the paper is to model synthetic social network based on relations obtained from the data collection about terroristic incidents to found new, often latent or unexpected characteristics and patterns of the data. The social network is constructed under the information obtained using two data mining techniques - association rules mining and clustering. Discovered relations based on the similar behavior were analyzed and visualized using the methods of graph theory. The latent ties in the social network were represented by the level of similarity of the objects, which was computed from the attribute analysis performed on the previous data mining level. 
For the analysis was used the Global Terrorism Database (GTD) produced by the National Consortium for the Study of Terrorism and Responses to Terrorism (START) ${ }^{1}$. There were published several works oriented to the visualization of the information obtained from GTD and some of them will be described in next chapter.

\section{Related work}

First analytical tool you probably meet while you start to interest in GTD is accessible on-line right on website of START and is called GTD Data Rivers (Lee, 2008). This web-based interactive exploratory tool provides aggregation of important variables from the database and visualization of results as comprehensible stack charts, what provides quick and easy insight to the data for experts with no need of downloading any special analytical software.

Next visualization system with focus on GTD offers more complex possibilities for visual explorations and it consists of three interlinked components with different complementary perspectives on the data - investigation, projection and exploration (Godwin, 2008), (Jones, 2008), (Kosara, 2006), (Wang, 2008). Illustration of these three components can be seen on Figure 1.

Figure 1: Three components of visualization system: Investigation, Projection and

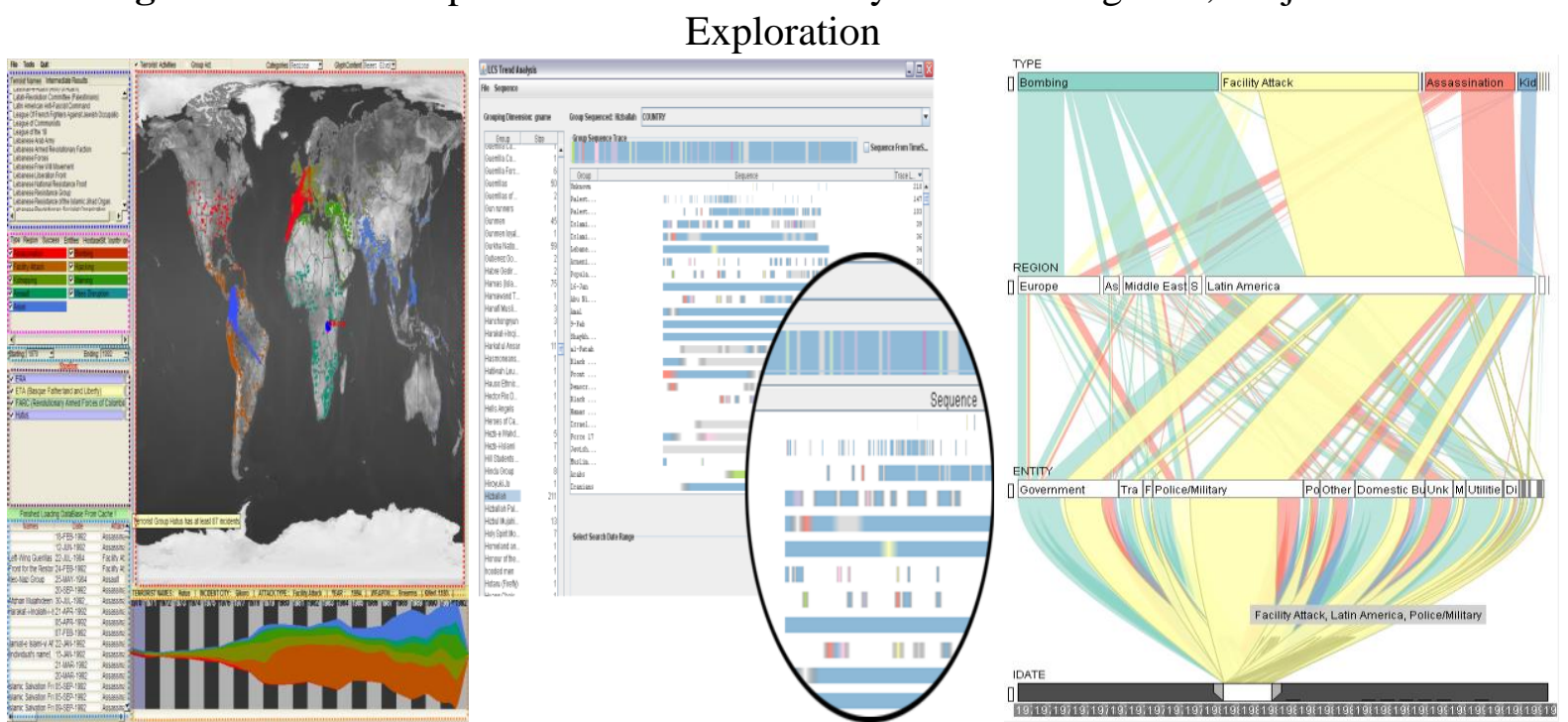

Source: Godwin (2008), Jones (2008), Kosara (2006), Wang (2008).

First component, (Jones, 2008), (Wang, 2008) ,offers Investigative view which is focused on investigation in geotemporal context built around basic questions who, what, when and where.

Second component, (Godwin, 2008), can be used to detailed investigation of temporal patterns and is based on methods successfully used in biometrics, particularly in longest common subsequence analysis of sequences of nucleotides. Methods for gene analysis were adjusted for data structure of GTD using sequences of attacks of terrorist groups instead of sequences in genes for comparison, so the component offers the comparison of the behavior of two logical groupings of terrorist's events as it changes over time - in this place we have to note that the term "behavior" has the quite different meaning that meaning as we use it in this work in the text below.

\footnotetext{
${ }^{1}$ Global Terrorism Database, START, accessed on 30 January 2011
} 
Third component (Kosara, 2006) supports exploration of relationships among dimensions in more abstract manner than previous two components. This component shows degree of correlation between categorical dimensions (attributes) using "ribbons" that are sized based on the number of cases two categories share. This component is very illustrative for smaller amount of categories and is very useful while focused on specific small subset of groups (categories), which we want to analyze. But in case of large amount of categories (in GTD there are for example more than thousand of different terroristic groups, hundreds of countries where attacks are committed), may happen to be almost impossible to get comprehensible visualization. It's also possible to explore with this tool relations among categories of more than two attributes (dimensions), but with multiplying number of categories from all dimensions it brings another difficulty in understanding of visualizations. To solve this problem with large amount of categories (in case of composition of multiple dimensions from data referred as "The Curse of Dimensionality" (Zhang, 2009)) we created method, that filter very effectively data preserving only interesting or important (captured by what we call "typical behavior" described later in text) relations in data, what (that interestingness or importance) can be defined by mean of some tunable parameters for filtering in selected data mining method. Filtered data are then presented graphically in synthetic social network for further visual investigation (you can see example on Figure 2).

Figure 2: Graph of Synthetic Social Network

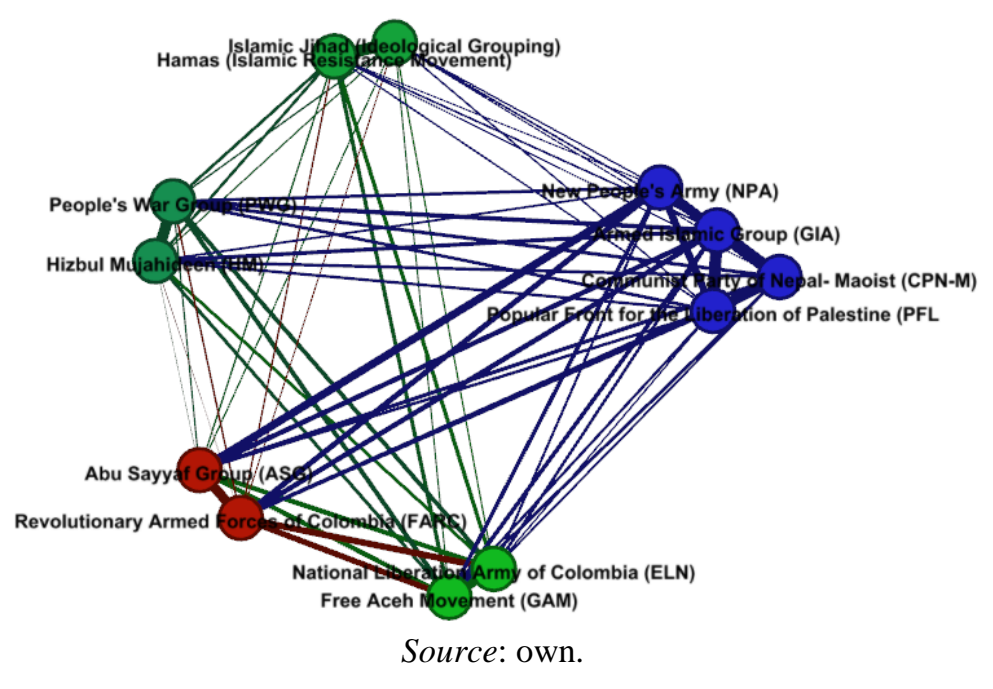

Moreover, using datamining paradigm that we want answers not only on what we have questioned but also what we have not - revealing interesting answers for questions which do not ordinarily come on mind, we can discover unexpected similarities among different terroristic groups. So, our method can serve in a first step for investigating interesting similarities among all terroristic groups in GTD and then in second step focusing on some community (group of terroristic groups) can be investigated more specifically similarities (or vice versa differences) among them by means of above mentioned methods.

\section{Typical behavior analysis}

Our intention is to extend visualization system mentioned in previous section with next complementary tool for visualization of all the information in GTD by means of what we call "typical behavior" of individual terroristic groups and present it graphically in synthetic social network, what can serve as first step of exploration of GTD in global scope and after this step, when we have analyzed interesting similarities among all terroristic groups in the data, we can continue with exploration of the data in more specific focus (as mentioned before). In the first 
subsection we formally define what we mean by the expression behavior and in the second subsection we discuss the mean of the adjective typical.

\subsection{Behavior}

Suppose we have data $\mathbf{D}(\boldsymbol{o}, \boldsymbol{X})$ in general form

Table 1: General form of data matrix

\begin{tabular}{|c|c|c|c|}
\hline & $X_{1}$ & $\ldots$ & $X_{n}$ \\
\hline$o^{(1)}$ & $x_{1}^{(1)}$ & $\ldots$ & $x_{n}^{(1)}$ \\
\hline$\vdots$ & $\vdots$ & $\vdots$ & $\vdots$ \\
\hline$o^{(j)}$ & $\ldots$ & $x_{i}^{(j)}$ & $\cdots$ \\
\hline$\vdots$ & $\vdots$ & $\vdots$ & $\vdots$ \\
\hline$o^{(m)}$ & $x_{1}^{(m)}$ & $\cdots$ & $x_{n}^{(m)}$ \\
\hline
\end{tabular}

Source: own.

where:

1) $\boldsymbol{o}=\left\{o^{(1)}, \ldots, o^{(m)}\right\}$ is set of some objects (processes, transactions, etc.),

2) $\boldsymbol{X}=\left\{X_{1}, \ldots, X_{n}\right\}$ is set of variables (attributes),

3) $x_{i}^{(j)}$ are values of $i$ th attribute measured on $j$ th object, formally ${ }^{2} X_{i}: \boldsymbol{o} \rightarrow \widehat{n}_{i}$ is mapping defined as $X_{i}\left(o^{(j)}\right)=x_{i}^{(j)}, \forall i \in \widehat{n}_{i}, \forall j \in \widehat{m}$ (we do not restrict $\widehat{n}_{i}$ to be a set of natural numbers - it can be any finite set of categories as every set of categories can be mapped to a set of natural number - we use $\widehat{n}_{i}$ just for simplification of notation) ${ }^{3}$.

This general form has also data from GTD, whose sample is illustrated in Table 2 and we use this sample for all examples in text below.

To form groups of objects which we want to compare among each other, we choose one attribute $X_{z}$ (let's call it $z$-attribute) from $\boldsymbol{X}$, which we use to separate objects from data $\mathbf{D}$ into classes. From previous chapter we assume that all attributes are mapping into finite sets of categories, so we can select $X_{z}$ arbitrarily. For example, we choose as $X_{z}$ attribute gname (from

), to which corresponds two categories from $\hat{n}_{z}$ ( $\{$ Hamas, Islamic Jihad $\}$ - let's call them $z-$ categories). Now we can split objects $\boldsymbol{o}$ to $n_{z}$ classes with conditions $X_{z}=\mathrm{cat}_{z}$, where cat $_{z} \in \widehat{n}_{z}$. So in our example now we have 2 groups of objects, where each corresponds to records about terroristic attacks for every individual terroristic group.

The rest of the attributes from $\boldsymbol{X}$, attributes $X_{i_{1}}, \ldots, X_{i_{k}}, \boldsymbol{i}=\left\{i_{1}, \ldots, i_{k}\right\}, \boldsymbol{i} \subset \hat{n}, k \in \hat{n}, z \notin \boldsymbol{i} \wedge z \in \hat{n}$, (let's call these attributes $i$-attributes), we use to create model of a group behavior based on frequency of occurrence of individual values of i-attributes of objects belonging to corresponding z-category. So in our example, we are interested how individual z-categories are related with values of the other attributes $X_{i_{1}}\left(=\right.$ country_txt) and $X_{i_{2}}$ ( = suicide), respectively to categories from $\hat{n}_{i_{1}}=\{$ Israel, Gaza Strip $\}$ and from $\hat{n}_{i_{2}}=\{0,1\}$ (let's call them $i$-categories). We can inspect these relations with questions "How many times Hamas attacked in Israel?", "How many times Hamas attacked in Gaza Strip?" or "How many times attacked Hamas by suicide attacker?", etc., by other words how are objects from individual z-

\footnotetext{
${ }^{2}$ We use notation $\widehat{n}=\{1, \ldots, n\}, n \in \mathbf{N}, \widehat{n} \subset \mathbf{N}$ (i.e. $\widehat{3}=\{1,2,3\}$ ).

${ }^{3}$ It implies that attributes with continuous (infinite) domain needs to be categorized before starting the process described in next chapters.
} 
categories distributed over i-categories. These numbers can be obtained by means of contingency table and with normalizing them by frequency of corresponding category from $\hat{n}_{z}$ (e.g. 10 for Hamas , 10 for Islamic Jihad) we get distribution of z-category over icategories. This distribution can be consequently compared with corresponding distributions of other z-categories.

Table 2: Data sample from GTD database

\begin{tabular}{|c|c|c|c|}
\hline & gname & country_txt & suicide \\
\hline 1 & Hamas & Israel & 0 \\
\hline 2 & Hamas & Israel & 0 \\
\hline 3 & Hamas & Israel & 0 \\
\hline 4 & Hamas & Israel & 1 \\
\hline 5 & Hamas & Israel & 1 \\
\hline 6 & Hamas & Gaza Strip & 0 \\
\hline 7 & Hamas & Gaza Strip & 0 \\
\hline 8 & Hamas & Gaza Strip & 0 \\
\hline 9 & Hamas & Gaza Strip & 0 \\
\hline 10 & Hamas & Gaza Strip & 0 \\
\hline 11 & Islamic Jihad & Israel & 0 \\
\hline 12 & Islamic Jihad & Israel & 0 \\
\hline 13 & Islamic Jihad & Israel & 0 \\
\hline 14 & Islamic Jihad & Israel & 0 \\
\hline 15 & Islamic Jihad & Gaza Strip & 0 \\
\hline 16 & Islamic Jihad & Gaza Strip & 1 \\
\hline 17 & Islamic Jihad & Gaza Strip & 0 \\
\hline 18 & Islamic Jihad & Gaza Strip & 0 \\
\hline 19 & Islamic Jihad & Gaza Strip & 1 \\
\hline 20 & Islamic Jihad & Gaza Strip & 1 \\
\hline
\end{tabular}

Source: own.

Generally speaking as "behavior" of z-category $c a t_{z} \in \widehat{n}_{z}$ we understand distribution of this zcategory over all i-categories. Formally:

\section{Definition 1:}

Let $i \in \hat{n}, z \in \hat{n}, i \neq z, \mathbf{D}(\boldsymbol{o}, \boldsymbol{X})$ are data in form of Table 1. Behavior of category cat $_{z} \in \widehat{n}_{z}$ over category cat $t_{i} \in \widehat{n}_{i}$ is mapping $B_{\mathbf{D}, X_{z}, X_{i}}: \widehat{n}_{z} \times \widehat{n}_{i} \rightarrow[0,1]$ defined as

$$
B_{\mathbf{D}, X_{z}, X_{i}}\left(c a t_{z}, c a t_{i}\right)=\frac{\left|X_{z}=c a t_{z} \wedge X_{i}=c a t_{i}\right|_{\mathbf{D}}}{\left|X_{z}=c a t_{z}\right|_{\mathbf{D}}}
$$

where $\mid$ Cond $\left.\right|_{\mathbf{D}}$ denotes number of objects from data $\mathbf{D}$ satisfying condition Cond.

From Table 2 we can compute behaviors $B$ for z-category Hamas

Table 3: Values of behavior for z-category Hamas

\begin{tabular}{|c|c|c|c|c|c|}
\hline$B_{\mathbf{D}, X_{z}, X_{i_{1}}}($ Hamas,Israel) & \multirow{2}{*}{$=$} & 0.5 & $B_{\mathbf{D}, X_{z}, X_{i_{2}}}($ Hamas, 0$)$ & \multirow{2}{*}{$=$} & 0.8 \\
\hline$B_{\mathbf{D}, X_{z}, X_{i_{1}}}($ Hamas,Gaza Strip) & & 0.5 & $B_{\mathbf{D}, X_{z}, X_{i_{2}}}($ Hamas, 1$)$ & & 0.2 \\
\hline
\end{tabular}

Source: own.

and for z-category Islamic Jihad.

Table 4: Values of behavior for Z-category Islamic Jihad

\begin{tabular}{|c|c|c|c|c|c|}
\hline$B_{\mathbf{D}, X_{z}, X_{i_{1}}}$ (Islamic Jihad,Israel) & \multirow{2}{*}{$=$} & 0.4 & $B_{\mathbf{D}, X_{z}, X_{i_{2}}}$ (Islamic Jihad, 0$)$ & \multirow{2}{*}{$=$} & 0.7 \\
\hline$B_{\mathbf{D}, X_{z}, X_{i_{1}}}$ (Islamic Jihad,Gaza Strip) & & 0.6 & $B_{\mathbf{D}, X_{z}, X_{i_{2}}}$ (Islamic Jihad,1) & & 0.3 \\
\hline
\end{tabular}


After comparing these behavior values for each z-category (we compare differences between values in pairs ( $B_{\mathbf{D}, X_{z}, X_{i_{1}}}$ (Hamas,Israel), $B_{\mathbf{D}, X_{z}, X_{i 1}}$ (Islamic Jihad,Israel)), ( $B_{\mathbf{D}, X_{z}, X_{i_{1}}}$ (Hamas, Gaza Strip), $B_{\mathbf{D}, X_{z}, X_{i_{1}}}$ (Islamic Jihad, Gaza Strip)), ( $B_{\mathbf{D}, X_{z}, X_{i_{2}}}$ (Hamas, 0), $B_{\mathbf{D}, X_{z}, X_{i_{2}}}$ (Islamic Jihad, 0)), etc., for all icategories) we can claim that behaviors of both z-categories are very similar (distributions of objects belonging to each z-category over i-attributes are almost equal (i.e. not bigger than $0.1)$ ). But this similarity is implied only when we compare z-categories over i-categories of one other i-attribute at once (e.g. we first compare behavior values $B_{\mathbf{D}, X_{z}, X_{i_{1}}}$ for Hamas with corresponding values $B_{\mathbf{D}, X_{z}, X_{i_{1}}}$ for Islamic Jihad, then we compare behavior values $B_{\mathbf{D}, X_{z}, X_{i_{2}}}$ for Hamas with corresponding values $B_{\mathbf{D}, X_{z}, X_{i_{2}}}$ for Islamic Jihad and so on in case there were more $\mathrm{i}$-attributes). But if we investigate data deeper, we can observe that $\mathrm{z}$ categories doesn't behave equal when both $X_{i_{1}}$ and $X_{i_{2}}$ are taken into account at one time. For example, 2 attacks out of 5 taken by Hamas in Israel were committed by suicide attacker, but for Islamic Jihad none of attacks in Israel was committed by suicide attacker. So, distributions of $z$-categories over Cartesian product of i-categories are quite different and to investigate these relations among categories of more than two attributes (i.e. among $X_{z}$ and more than one i-attribute) we formally define behavior of z-category $c a t_{z} \in \hat{n}_{z}$ over set of icategories cat $_{i_{1}} \in n_{i_{1}}, \ldots$, cat $_{i_{k}} \in n_{i_{k}}$ as:

\section{Definition 2:}

Let $\boldsymbol{i}=\left\{i_{1}, \ldots, i_{k}\right\}, \boldsymbol{i} \subset \hat{n}, k \in \hat{n}, \quad z \notin \boldsymbol{i} \wedge z \in \hat{n}, \mathbf{D}$ are data in form of Table 1. Behavior of category cat $t_{z} \in \widehat{n}_{z}$ over categories cat $_{i_{1}} \in \widehat{n}_{i_{1}}, \ldots$, cat $_{i_{k}} \in \widehat{n}_{i_{k}}$ is mapping

$$
B_{\mathbf{D}, X_{z}, X_{i_{1}}, \ldots, X_{i_{k}}}: \widehat{n}_{z} \times \widehat{n}_{i_{1}} \times \ldots \times \widehat{n}_{i_{k}} \rightarrow[0,1]
$$

defined as

$$
B_{\mathbf{D}, X_{z}, X_{i_{1}}, \ldots, X_{i_{k}}}\left(\text { cat }_{z}, \text { cat }_{i_{1}}, \ldots, c a t_{i_{k}}\right)=\frac{\left|X_{z}=c a t_{z} \wedge X_{i_{1}}=c a t_{i_{1}} \wedge \ldots \wedge X_{i_{k}}=c a t_{i_{k}}\right|_{\mathbf{D}}}{\left|X_{z}=c a t_{z}\right|_{\mathbf{D}}}
$$

where $\mid$ Cond $\left.\right|_{\mathbf{D}}$ has the same meaning as in Definition 1.

With this definition we can compute behavior of z-categories over Cartesian product of icategories $\left(\hat{n}_{i_{1}} \times \widehat{n}_{i_{2}}\right)$ which is now in form of matrix.

Table 5: Behavior values for Hamas on both i-categories

\begin{tabular}{|l|l|l|c|c|}
\hline$B_{\mathbf{D}, X_{z}, X_{i_{1}}, X_{i_{2}}}$ (Hamas,Israel,0) & $B_{\mathbf{D}, X_{z}, X_{i_{1}}, X_{i_{2}}}$ (Hamas, Gaza Strip ,0) \\
\hline$B_{\mathbf{D}, X_{z}, X_{i_{1}}, X_{i_{2}}}$ (Hamas, Israel,1) & $B_{\mathbf{D}, X_{z}, X_{i_{1}}, X_{i_{2}}}$ (Hamas, Gaza Strip,1) & $=0.3$ & 0.5 \\
\cline { 1 - 5 } & 0.2 & 0 \\
\hline
\end{tabular}

Source: own.

Table 6: Behavior values for Islamic Jihad on both i-categories

\begin{tabular}{|l|l|l|c|c|}
\hline$B_{\mathbf{D}, X_{z}, X_{i_{1}}, X_{i_{2}}}$ (Islamic Jihad, Israel,0) & $B_{\mathbf{D}, X_{z}, X_{i_{1}}, X_{i_{2}}}$ (Islamic Jihad, Gaza Strip ,0) \\
\hline$B_{\mathbf{D}, X_{z}, X_{i_{1}}, X_{i_{2}}}$ (Islamic Jihad, Israel, 1) & $B_{\mathbf{D}, X_{z}, X_{i_{1}}, X_{i_{2}}}$ (Islamic Jihad, Gaza Strip,1)
\end{tabular}$=$\begin{tabular}{c|c|c|}
0.4 & 0.3 \\
\hline
\end{tabular}

Source: own. 
From these matrices can be seen that distribution for individual z-categories over Cartesian product of all sets of $\mathrm{i}$-categories differs even if both $\mathrm{z}$-categories behave almost equally over sets of categories $\hat{n}_{i_{1}}, \hat{n}_{i_{2}}$ taken separately. This is implying that to compare properly behavior of z-categories we would need to compute not only behavior over all sets of i-categories separately, but also we would have to compute behavior of z-categories over Cartesian products of all possible combinations of $\operatorname{sets} \widehat{n}_{i_{1}}, \ldots, \hat{n}_{i_{k}}$. For one z-category and one set of cardinality of $k$ i-attributes we can compute $\prod_{l=1}^{k} n_{i_{l}}$ values of $B_{\mathbf{D}, X_{z}, X_{i_{1}}, \ldots, X_{i_{k}}}$ and for every $k \leq n-1$ we can combine $\left(\begin{array}{c}n-1 \\ k\end{array}\right)$ sets of $\mathrm{i}$-attributes from set of all i-attributes. From these values we can construct behavioral vector in form:

\section{Definition 3:}

Let $z \in \widehat{n}$, cat $_{z} \in \widehat{n}_{z}$. Behavioral vector is vector $\vec{B}_{\text {cat }} \in[0,1]^{\# B_{z}(\mathbf{D})}$ in form

$$
\begin{aligned}
& \vec{B}_{c a t_{z}}=\left(B_{\mathbf{D}, X_{z}, X_{i_{1}}}\left(c a t_{z}, 1\right), \ldots, B_{\mathbf{D}, X_{z}, X_{i_{1}}}\left(c a t_{z}, n_{i_{1}}\right), B_{\mathbf{D}, X_{z}, X_{i_{2}}}\left(c a t_{z}, 1\right), \ldots, B_{\mathbf{D}, X_{z}, X_{i_{2}}}\left(c a t_{z}, n_{i_{2}}\right), \ldots,\right. \\
& B_{\mathbf{D}, X_{z}, X_{i_{k}}}\left(\text { cat }_{z}, 1\right), \ldots, B_{\mathbf{D}, X_{z}, X_{i_{k}}}\left(c a t_{z}, n_{i_{k}}\right), B_{\mathbf{D}, X_{z}, X_{i_{1}}, X_{i_{2}}}\left(\text { cat }_{z}, 1,1\right), B_{\mathbf{D}, X_{z}, X_{i_{1}}, X_{i_{2}}}\left(\text { cat }_{z}, 1,2\right), \ldots, \\
& B_{\mathbf{D}, X_{z}, X_{i_{1}}, X_{i_{2}}}\left(c a t_{z}, n_{i_{1}}, n_{i_{2}}\right), \ldots, B_{\mathbf{D}, X_{z}, X_{i_{k-1}}, X_{i_{k}}}\left(c a t_{z}, 1,1\right), \ldots, B_{\mathbf{D}, X_{z}, X_{i_{k-1}}, X_{i_{k}}}\left(\text { cat }_{z}, n_{i_{k_{k-1}}}, n_{i_{k}}\right), \ldots, \\
& \text { (all possible triplets of } i \text {-attributes and } i \text {-categories), } \\
& \text {..., } \\
& \text { (all possible }(k-1) \text {-tuplets of } i \text {-attributes and } i \text {-categories), } \\
& \left.B_{\mathbf{D}, X_{z}, X_{i_{1}}, \ldots, X_{i_{k}}}\left(c a t_{z}, 1, \ldots, 1\right), \ldots, B_{\mathbf{D}, X_{z}, X_{i_{1}}, \ldots, X_{i_{k}}}\left(\text { cat }_{z}, n_{i_{1}}, \ldots, n_{i_{k}}\right)\right)^{\mathrm{T}}
\end{aligned}
$$

where $\# B_{z}(\mathbf{D})$ express size of $\vec{B}_{\text {cat }}$ (is the same for every cat $t_{z} \in \widehat{n}_{z}$ ) as

$$
\# B_{z}(\mathbf{D})=\sum_{k=1}^{n-1} \sum_{\left(i_{1}, \ldots, i_{k}\right) \in\left(\begin{array}{l}
n \\
k
\end{array}\right) \wedge(\forall j \in \widehat{k})\left(i_{j} \neq z\right)} \prod_{l=1}^{k} n_{i_{l}}
$$

where $\overline{\left(\begin{array}{l}n \\ k\end{array}\right)}=$ all $k$-tuples from $\hat{n}, k \in \hat{n}$ (i.e. $\overline{\left(\begin{array}{l}3 \\ 2\end{array}\right)}=\{\{1,2\},\{1,3\},\{2,3\}\}$ ).

So in our example we have $\vec{B}_{\text {Hamas }}=(0.5,0.5,0.8,0.2,0.2,0.3,0.5,0)^{\mathrm{T}}$ and $\vec{B}_{\text {IslamicJihad }}=(0.4$, $0.6,0.7,0.3,0.4,0,0.3,0.3)^{\mathrm{T}}$.

It can be seen, that size of $\vec{B}_{\text {cat }}$ grows very rapidly while $n$ and $n_{i}$ 's are growing and it is neither very efficient nor necessary to compute all values of behavior to compare individual zcategories. More efficient way to compute only "typical" elements of behavior is discussed in next section.

\subsection{Association rules}

Even if term association rules was strongly popularized by Agrawal in 1990s with discovering relations in transactional databases (and this is the reason why we use this widely known term) (Agrawal, 1993), there was strong effort in this area two decades before while studying Boolean predicate logic in framework of so-called observation logic which includes also association rules as a one type of rules with generalized quantifier (Hájek, 1987). This framework underlies one of the earliest methods for the extraction of general rules from data, called General Unary Hypotheses Automaton (GUHA) (Hájek, 1966). 
There exists many generalized quantifiers defined in GUHA method (see (Hájek, 1987) for formal definition), but we will describe only one quantifier called founded implication, which belongs to class of so called implicational quantifiers (Hájek, 1987). This quantifier (as the rest of generalized quantifiers) is defined by means of $\{0,1\}$-valued function on values of socalled 4-fold table. As rules in GUHA have always the form Ant $\sim$ Suc, where Ant and Suc are Boolean predicates and $\sim$ is binary generalized quantifier, 4-fold table has form

Table 7: 4-fold table

\begin{tabular}{|l|l|l|}
\hline & Suc & $\neg$ Suc \\
\hline Ant & $a$ & $b$ \\
\hline$\neg$ Ant & c & $d$ \\
\hline \multicolumn{3}{|c|}{ Source: own. }
\end{tabular}

where $\quad a=|A n t \wedge S u c|_{\mathbf{D}}, b=|A n t \wedge \neg S u c|_{\mathbf{D}}, c=|\neg A n t \wedge S u c|_{\mathbf{D}}, \quad d=|\neg A n t \wedge \neg S u c|_{\mathbf{D}} . \quad$ Objects from data $\mathbf{D}$ are separated into 4 folds (from this fact is derived the name of the table), and we can observe that $a+b+c+d=m$ always holds for data $\mathbf{D}$. Validity of rule with generalized quantifier $\sim$ is than determined by value of truth function $\mathrm{Tf}_{\sim}$, which is mapping

$$
\mathrm{Tf}_{\sim}: \mathrm{N}_{0}^{4} \rightarrow\{0,1\}
$$

related with that quantifier $\sim$. Rule with quantifier $\sim$ is then valid if $\operatorname{Tf}_{\sim}(a, b, c, d)=1$ and not valid if $\operatorname{Tf}_{\sim}(a, b, c, d)=0$.

As written above we only describe one quantifier called founded implication noted as $\Rightarrow_{s, \theta}, s, \theta \in(0,1]$. This quantifier is valid exactly for those data for which the conditional probability $p(S u c \mid A n t)$ of validity of $S u c$ conditioned on Ant, estimated with the unbiased estimate $\frac{a}{a+b}$ is at least $\theta$, whereas Ant and Suc are simultaneously valid at least the proportion $s$ of the data (Hájek, 1987). Hence, truth function $\mathrm{Tf}_{\Rightarrow_{s, \theta}}$ for founded implication quantifier in rule $A n t \Rightarrow_{s, \theta} S u c$ is defined as

$$
\begin{aligned}
& \left\lceil 1 \text { iff } \frac{a}{a+b} \geq \theta \wedge \frac{a}{a+b+c+d} \geq s\right. \\
& \operatorname{Tf}_{\Rightarrow_{s, \theta}}(a, b, c, d)=\{ \\
& \text { (0 else. }
\end{aligned}
$$

As behavior $B_{\mathbf{D}, X_{z}, X_{i_{1}}, \ldots, X_{i_{k}}}\left(\right.$ cat $_{z}$, cat $_{i_{1}}, \ldots$, cat $\left._{i_{k}}\right)$ is defined by (2) we can see that formula $X_{z}=c a t_{z} \Rightarrow_{s, \theta} X_{i_{l}}=c a t_{i_{l}} \wedge \ldots \wedge X_{i_{k}}=$ cat $_{i_{k}}$ is valid, if and only if $B_{\mathbf{D}, X_{z}, X_{i_{1}}, \ldots, X_{i_{k}}}\left(c a t_{z}\right.$, cat $\left._{i_{1}}, \ldots, c a t_{i_{k}}\right)=\frac{\left|X_{z}=c a t_{z} \wedge X_{i_{1}}=c a t_{i_{1}} \wedge \ldots \wedge X_{i_{k}}=c a t_{i_{k}}\right|_{\mathbf{D}}}{\left|X_{z}=c a t_{z}\right|_{\mathbf{D}}} \geq \theta$ and $\frac{\mid X_{z}=\text { cat }_{z} \wedge X_{i_{1}}=\text { cat }_{i_{1}} \wedge \ldots \wedge X_{i_{k}}=\text { cat }_{i_{k}} \mid}{m} \geq s$.

We can use this important relation between value of $B_{\mathbf{D}, X_{z}, X_{i_{1}}, \ldots, X_{i_{k}}}\left(\right.$ cat $_{z}$, cat $_{i_{1}}, \ldots$, cat $\left._{i_{k}}\right)$ and validity of rule in form of $X_{z}=c a t_{z} \Rightarrow_{s, \theta} X_{i_{l}}=c a t_{i_{l}} \wedge \ldots \wedge X_{i_{k}}=c a t_{i_{k}}$ with quantifier $\Rightarrow_{s, \theta}$ to filter very efficiently amount of values of $B$ which we will compute, by setting parameters $s$ 
and $\theta$, and also we solve the problem of extremely large amount of values determined by $\# B_{z}(\mathbf{D})$.

As we see from definition of behavioral vector (3), there is just one corresponding formula for each element $j$ of $\vec{B}_{\text {cat }_{z}}$ for $k$-tuples of i-attributes $X_{i_{1}}, \ldots, X_{i_{k}}$ and i-categories cat $_{z}$, cat $_{i_{1}}, \ldots$, cat $_{i_{k}}$, so we denote it as

$$
\text { formula }\left(\left(\vec{B}_{c a t_{z}}\right)_{j}\right)=\left(X_{z}=c a t_{z} \Rightarrow_{s, \theta} X_{i_{l}}=c a t_{i_{l}} \wedge \ldots \wedge X_{i_{k}}=c a t_{i_{k}}\right) .
$$

Together with this we denote

$$
\operatorname{Tf}_{\Rightarrow_{s, \theta}}\left(\left(\vec{B}_{c a t_{z}}\right)_{j}\right)=\operatorname{Tf}_{\Rightarrow_{s, \theta}}(a, b, c, d)
$$

for formula formula $\left(\left(\vec{B}_{c a t_{z}}\right)_{j}\right)$. We construct set of indexes $J$

$$
J=\left\{j \in \mathbf{N} \mid\left(\exists c a t_{z} \in \widehat{n}_{z}\right)\left(\text { formula }\left(\left(\vec{B}_{\text {cat }_{z}}\right)_{j}\right) \text { is valid for data } \mathbf{D}\right)\right\}
$$

and then we can construct vector of reduced size, which we call typical behavior vector denoted as ${ }_{\text {Typical }} \vec{B}_{\text {cat }}$ and which is constructed only from selected (by set of indexes $J$ ) values of $\vec{B}_{\text {cat }}$ as:

\section{Definition 4:}

Let $z \in \hat{n}$, cat $_{z} \in \widehat{n}_{z}, s, \theta \in(0,1], \vec{B}_{\text {cat }}$ is behavioral vector (from Def. 3) and symbols $J$ and $\operatorname{Tf}_{\Rightarrow_{s, \theta}}\left(\left(\vec{B}_{\text {cat }_{z}}\right)_{j}\right)$ have meaning from (8) and (7), respectively. Then typical behavior vector for cat $_{z}$ (noted ${ }_{\text {Typical }} \vec{B}_{\text {cat }}$ ) is defined as

$$
\left({ }_{\text {Typical }} \vec{B}_{\text {cat }}\right)_{l}=\left(\vec{B}_{\text {cat }}\right)_{J_{l}} \cdot \mathrm{Tf}_{\Rightarrow_{s, \theta}}\left(\left(\vec{B}_{c a t_{z}}\right)_{j}\right), \forall l=1, \ldots, \# J
$$

where \#J denotes cardinality of set $J$. Vector ${ }_{\text {Typical }} \vec{B}_{\text {cat }}$ is of course $s$ and $\theta$ dependent, but to not overcomplicate notation we omit it.

So general process of construction of ${ }_{\text {Typical }} \vec{B}_{\text {cat }}$ consists of three steps:

1. extraction of all valid rules in form $X_{z}=c a t_{z} \Rightarrow_{s, \theta} X_{i_{l}}=c a t_{i_{l}} \wedge \ldots \wedge X_{i_{k}}=c a t_{i_{k}}$ and to each rule compute corresponding behavior value $B_{\mathbf{D}, X_{z}, X_{i}, \ldots, X_{i_{k}}}\left(\right.$ cat $_{z}$, cat $\left._{i_{1}}, \ldots, c a t_{i_{k}}\right)$,

2. construction of set of indexes $J(8)$,

3. construction of ${ }_{\text {Typical }} \vec{B}_{\text {cat }}$ according to Definition 4 .

To clarify more the process of construction of typical behavior vector, let's look on our example. First step is to set values of $s$ and $\theta$. By setting those two parameters we define the meaning of term "typical" behavior. By setting parameter $s$ closer to 1 we filter low frequented combinations of all categories (z-category and i-categories) and with setting parameter $\theta$ closer to 1 we filter low values of behavior $B$, focusing rather on values 
(combinations of $\mathrm{i}$-categories) that are frequent for individual $\mathrm{z}$-category than on less frequented combinations of i-categories. So setting of values $s$ and $\theta$ depend on data we analyze and we have to realize which frequencies of occurrence categories in data we still consider as significant and which rather as random or accidental. For our example let's set $s=$ 0.1 and $\theta=0.3$. Then we generate set of 12 rules

$$
\begin{aligned}
& X_{z}=\text { Hamas } \Rightarrow_{0.1,0.3} X_{i_{l}}=\text { Israel } \\
& X_{z}=\text { Hamas } \Rightarrow_{0.1,0.3} X_{i_{l}}=\text { Gaza Strip } \\
& X_{z}=\text { Hamas } \Rightarrow_{0.1,0.3} X_{i_{2}}=0 \\
& X_{z}=\text { Hamas } \Rightarrow_{0.1,0.3} X_{i_{1}}=\text { Israel } \wedge X_{i_{2}}=0 \\
& X_{z}=\text { Hamas } \Rightarrow_{0.1,0.3} X_{i_{1}}=\text { Gaza Strip } \wedge X_{i_{2}}=0 \\
& X_{z}=\text { Islamic Jihad } \Rightarrow_{0.1,0.3} X_{i_{l}}=\text { Israel } \\
& X_{z}=\text { Islamic Jihad } \Rightarrow_{0.1,0.3} X_{i_{l}}=\text { Gaza Strip } \\
& X_{z}=\text { Islamic Jihad } \Rightarrow_{0.1,0.3} X_{i_{2}}=0 \\
& X_{z}=\text { Islamic Jihad } \Rightarrow_{0.1,0.3} X_{i_{2}}=1 \\
& X_{z}=\text { Islamic Jihad } \Rightarrow_{0.1,0.3} X_{i_{1}}=\text { Israel } \wedge X_{i_{2}}=0 \\
& X_{z}=\text { Islamic Jihad } \Rightarrow_{0.1,0.3} X_{i_{1}}=\text { Gaza Strip } \wedge X_{i_{2}}=0 \\
& X_{z}=\text { Islamic Jihad } \Rightarrow_{0.1,0.3} X_{i_{1}}=\text { Gaza Strip } \wedge X_{i_{2}}=0
\end{aligned}
$$

and to each rule we compute corresponding value of $B$ (from Def. 2). So set of indexes $J$ is now $\{1,2,3,4,6,7,8\}$ and then ${ }_{\text {Typical }} \vec{B}_{\text {Hamas }}=(0.5,0.5,0.8, \mathbf{0}, 0.3,0.5,0)^{\mathrm{T}}$ and Typical $\vec{B}_{\text {IslamicJihad }}=(0.4,0.6,0.7,0.3,0.4,0.3,0.3)^{\mathrm{T}}$, so every fifth element from $\vec{B}_{\text {cat }}$ is omitted and bold-marketed value is value, where $B$ value is replaced with 0 because of invalidity of formula $\left(\left(\vec{B}_{\text {Hamas }}\right)_{5}\right)$.

Last but not least note for Def. 4 (which also clarify Step 3 from process of construction of $\left.{ }_{\text {Typical }} \vec{B}_{\text {cat }}\right)$ is on use of $\operatorname{Tf}_{\Rightarrow_{s, \theta}}\left(\left(\vec{B}_{\text {cat }}\right)_{j}\right)$ in definition of ${ }_{T_{y p i c a l}} \vec{B}_{\text {cat }}$ - using this multiplying value we avoid the need of using data $\mathbf{D}$ one more time to compute this typical behavior vector. Realize that we used data $\mathbf{D}$ to extract all valid association rules in form of $X_{z}=c a t_{z} \Rightarrow_{s, \theta} X_{i_{l}}=c a t_{i_{l}} \wedge \ldots \wedge X_{i_{k}}=$ cat $_{i_{k}}$ while compute to each rule corresponding value of $B$. If we would like to compute all values from $\vec{B}_{c a t_{z}}$ with indexes in $J$, we would have to use data for the second time. Instead of this we use only values of $\left(\vec{B}_{\text {cat }}\right)_{j}$ for valid formula $\left(\left(\vec{B}_{\text {cat }_{z}}\right)_{j}\right)$ while in case of invalidity of formula $\left(\left(\vec{B}_{c a t_{z}}\right)_{j}\right)$ we replace $\left(\vec{B}_{c a t_{z}}\right)_{j}$ with 0 . Our experiments showed that this approach does not bring any significant discrepancies in final results.

With typical behavior of z-categories cat $_{z} \in \widehat{n}_{z}$ captured by vectors ${ }_{\text {Typical }} \vec{B}_{\text {cat }}$ we can now step to visualization level, where we model so-called synthetic social network. 


\section{Social network model}

A social network ( $\mathrm{SN}$ ) is typically a set of people or groups of people with similar pattern of contacts or interactions such as friendship, co-working, or information exchange (Wasserman, 1994). Social networks are usually represented using graph theory (with graphs), where nodes represent individuals or groups and lines represent relations among them (Carrington, 2005). These graphs can be directed or undirected, depending on the type of the relation between the linked nodes. To designate different interaction strengths, there can be assigned weights to the links (edges) between the nodes. Using other additional information (often not directly related to the interactions, e.g. behavior), we can construct synthetic social networks, where the relations between the nodes can be represented by the similarity of this kind of information (e.g. similar typical behavior).

\subsection{Computing similarities and visualization of social network model}

From the previous data mining level related to the extraction of the association rules, we have constructed set of vectors ${ }_{\text {Typical }} \vec{B}_{\text {cat }}$ for every Z-category cat $_{z} \in \widehat{n}_{z}$ (Def. 4). Consequently from this set of $n_{z}$ behavioral vectors we have created Similarity matrix $S=[0,1]^{\hat{n}_{z} \times \bar{n}_{z}}$ using Cosine measure for computing the similarity $s_{i, j}$ between two objects $o_{i} \in \widehat{n}_{z}$ and $o_{j} \in \widehat{n}_{z}$ (terrorist groups)(Han, 2006):

$$
s_{i, j}=\frac{\sum_{k=1}^{\# J}\left(\left(_{\text {Typical }} \vec{B}_{i}\right)_{k}\left({ }_{\text {Typical }} \vec{B}_{j}\right)_{k}\right.}{\sqrt{\sum_{k=1}^{\# J}\left(\left(_{\text {Typical }} \vec{B}_{i}\right)_{k}\right)^{2}} \sqrt{\sum_{k=1}^{\# J}\left(\left(_{\text {Typical }} \vec{B}_{j}\right)_{k}\right)^{2}}}
$$

for $\forall i \in \widehat{n}_{z}, \forall j \in \widehat{n}_{z}$.

Figure 3: Synthetic Social Network based on 12 i-attributes

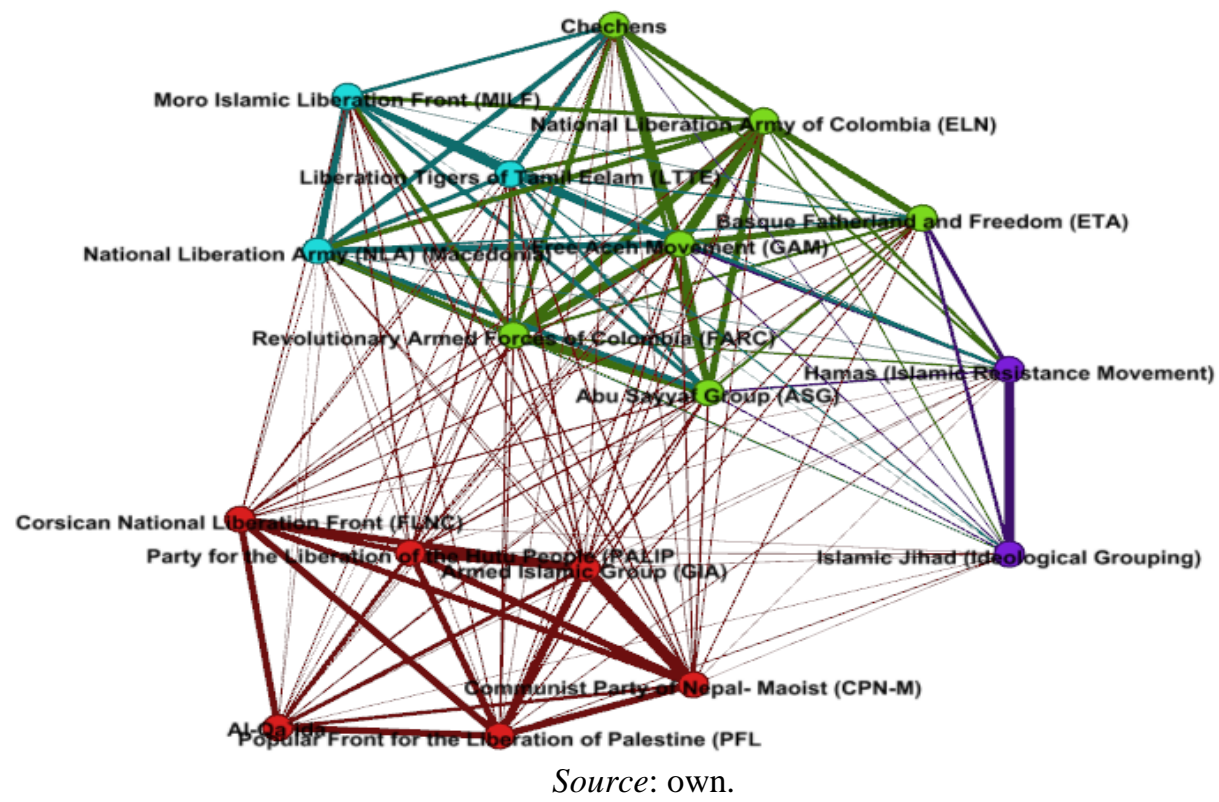

Model of the synthetic social network was obtained using principles of graph theory. The model was constructed with the undirected weighted graph $G=(O, E)$ where $O$ is set of 
objects (terrorist groups) from the Similarity matrix and $E$ is set of edges, which represent relations between them. The weight of the edges is defined using the similarity measure $s_{i, j}$.

\section{Experiments}

The initial graph contained 33 nodes with 528 edges (which represents one large connected component). For discovering significant communities with similar behavior we processed the edge filtering and graph partitioning based on the selected level of edge weight $>0.6$. For the visualization of the SN model was used the algorithm Force Atlas. The visualization was provided for both experiments with different number of selected attributes characterizing the similar behavior of the terrorist groups (see section 2.2). In the Table 7 there are presented parameters of both obtained graphs after the filtering and the graph partitioning.

Table 8: Graph Parameters

\begin{tabular}{|l|c|c|}
\hline & Experiment 1 & Experiment 2 \\
\hline i-Attributes & 8 & 12 \\
\hline Extracted rules & 2005 & 9252 \\
\hline Nodes before filtering & 33 & 33 \\
\hline Edges before filtering & 528 & 528 \\
\hline Nodes after filtering & $12(36,36 \%)$ & $17(51,52 \%)$ \\
\hline Edges after filtering & $66(12,5 \%)$ & $136(26,73 \%)$ \\
\hline Number of communities & 5 & 4 \\
\hline Modularity & 0.6875 & 0.594 \\
\hline \multicolumn{2}{|c|}{ Source: own. }
\end{tabular}

On the figures 2 and 3 we can see the visual representation of the models of synthetic social networks constructed from the GDT database for the year 2001 using the open-source software Gephi. The first graph (Figure 2) was obtained for the 8 selected i-attributes, the second (Figure 3 ) for 12 i-attributes. Both graphs show communities of terrorist groups with similar behavior. The width of the edges represents the strength of the relation (based on value of similarity) between terrorist groups. From both graph we can see, that the community detection can be influenced by the selection of appropriate attributes (which can depend on the user requirements).

\section{Conclusion}

The paper is oriented to the modeling of the synthetic social network based on the similar behavior of the terrorist groups extracted from the data collection GDT. The expression of typical behavior was formalized using the association rules mining method; the similarity between the terrorist groups based on their typical behavior was then analyzed and visualized using the methods of the graph theory. As an example there were processed two experiments with the different amount of selected attributes and two models of the synthetic social network were presented. The obtained graphs showed that the proposed data mining method is effective and can facilitate the orientation in the large amount of the extracted association rules. The extracted information can be applied in the prediction process or for construction of classification models using the relations within the data records or between the data collections. This type of information can be valuable in the decision making process and forecast the effects of those decisions. The proposed model then can be helpful in the identification process of the attacker or known terrorist group in the situations, when incident occurs. Also, it must be noted that the proposed approach involves rich parametric configuration and thus, for a practical use, there will be required the knowledge of domain experts. In future work we intent to visualize the evolution of the obtained synthetic social network model. 


\section{References}

[1] AGRAWAL, R., T. IMIELINSKI and A. SWAMI, 1993. Mining association rules between sets of items in large databases. In Proc. 1993 ACM-SIGMOD Int. Conf. Management of Data (SIGMOD'93), Washington, DC, pp. 207-216.

[2] BENOÎT, G., 2002. Data Mining. Annual Review of Information Science and Technology. Vol. 36, pp 265-310.

[3] CARRINGTON P. J., J. SCOTT and S. WASSERMAN, 2005. Models and Methods in Social Network Analysis. Cambridge: Cambridge University Press.

[4] FREEMAN, L. C., 2004. The Development of Social Network Analysis: A Study in the Sociology of Science. Empirical Press.

[5] GODWIN, A., R. CHANG, R. KOSARA and W. RIBARSKY, 2008. Visual analysis of entity relationships in global terrorism database. In SPIE Defense and Security Symposium.

[6] HÁJEK, P. and T. HAVRÁNEK, 1987. Mechanizing Hypothesis Formation. Springer Verlag, Berlin.

[7] HAN, J. and M. KAMBER, 2006. Data Mining Concepts and Techniques. Elsevier Science Ltd., pp 745. ISBN 1-55860-901-6.

[8] HAND, D. J., P. SMYTH and H. MANILLA. Principles of Data Mining. MIT Press, 2001. ISSN 0-262-08290-X.

[9] LAFREE, G. and L. DUGAN, 2007. "Global Terrorism Database 1970-1997", [Computer file]. ICPSR04586-v1. College Park, MD: University of Maryland [producer], 2006. Ann Arbor, MI: Inter-university Consortium for Political and Social Research [distributor].

[10] LEE, J., 2008. Exploring Global Terrorism Data: A Web-based Visualization of Temporal Data. In ACM Crossroads, Vol. 15, (2), pp. 7-16.

[11] ŠIMŮNEK, M., 2003. Academic KDD project LISP-Miner. In A. Abraham, K. Franke, K. Koppen (Eds.), Advances in Soft Computing - Systems Design and Applications. Springer Verlag, Heidelberg, pp. 263-272.

[12] WASSERMAN, S. and K. FAUST, 1994. Social Network Analysis. Cambridge: Cambridge University Press.

[13] P. HAJEK, I. HAVEL and M. CHYTIL, 1996: The GUHA method of automatic hypotheses determination. Computing 1, fasc. 4, 293-308.

[14] JONES, J., R. CHANG, T. BUTKIEWICZ and W. RIBARSKY, 2008. Visualizing uncertainty for geographical information in Global Terrorism Database. In SPIE Defense and Security Symposium.

[15] KOSARA, R., F. BENDIX and H. HAUSER, 2006. Parallel sets: visual analysis of categorical data. IEEE Transactions on Visualization and Computer Graphics 12(4):558-564.

[16] WANG, X., E. MILLER, K. SMARICK, W. RIBARSKY and R. CHANG, 2008. Investigative visual analysis of Global Terrorism Database. In Journal of Computer Graphics Forum. Volume 27, Issue 3, pages 919-926, May 2008.

[17] ZHANG, H., B. CLARK and E. FOKOUÉ, 2009. Principles and theory for Data Mining and Machine Learning. Springer. 